Canadian

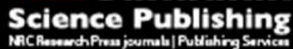

Applied Physiology, Nutrition, and Metabolism Physiologie appliquée, nutrition et métabolisme

\title{
L-carnitine supplement reduces skeletal muscle atrophy induced by prolonged hindlimb suspension in rats
}

\begin{tabular}{|r|l|}
\hline Journal: & Applied Physiology, Nutrition, and Metabolism \\
\hline Manuscript ID & apnm-2016-0094.R1 \\
\hline Danuscript Type: & Article \\
\hline Complete List of Authors: & $\begin{array}{l}\text { Jang, Jiwoong; Konkuk University, Department of Pysical Education } \\
\text { Park, Jonghoon; Korea University, Department of Pysical Education } \\
\text { Chang, Hyukki; Seoul Women\'s University, Department of Human } \\
\text { Movement Science } \\
\text { Lim, Kiwon; Konkuk University, Department of Pysical Education }\end{array}$ \\
\hline Keyword: & $\begin{array}{l}\text { weightless, hindlimb suspension, muscle atrophy, L-carnitine, muscle- } \\
\text { specific ubiquitin E3 ligases }\end{array}$ \\
\hline \multicolumn{2}{|c}{} \\
\hline
\end{tabular}

SCHOLARONE ${ }^{m}$

Manuscripts 
L-carnitine supplement reduces skeletal muscle atrophy induced by prolonged hindlimb suspension in rats

Jiwoong Jang ${ }^{1}$, Jonghoon Park ${ }^{2}$, Hyukki Chang ${ }^{3}$, Kiwon Lim ${ }^{1 *}$

${ }^{1}$ Laboratory of Exercise Nutrition, Department of Physical Education, Konkuk University, Seoul,

Korea, ${ }^{2}$ Department of Physical Education, Korea University, Seoul, Korea, ${ }^{3}$ Department of Human Movement Science, Seoul Women's University, Seoul, Korea.

\section{Correspondence Author:}

Kiwon Lim, Ph.D.

Laboratory of Exercise Nutrition,

Department of Physical Education, Konkuk University,

120, Neungdong-ro, Gwangjin-gu, Seoul 143-701, Korea

Tel: +82-2-450-3827/ Fax +82-452-6027

E-mail: $\underline{\text { exercise@,konkuk.ac.kr }}$

Running Title: Effects of L-carnitine on muscle atrophy induced by hindlimb suspension 


\section{Abstract}

L-carnitine was recently found to down-regulate the ubiquitin proteasome pathway (UPP) and increase IGF-1 concentrations in animal models. However, the effect of L-carnitine administration on disuse muscle atrophy induced by hindlimb suspension has not yet been studied. Thus, we hypothesized that L-carnitine may have a protective effect on muscle atrophy induced by hindlimb suspension via the Akt1/mTOR and or UPP. Male Wistar rats were assigned to 3 groups: hindlimb suspension group, hindlimb suspension with L-carnitine administration $\left(1250 \mathrm{mg} \cdot \mathrm{kg}^{-1} \cdot \mathrm{day}^{-1}\right)$ group, and pair-fed group adjusted hindlimb suspension. L-carnitine administration for 2 weeks of hindlimb suspension alleviated the decrease in weight and fiber size in the soleus muscle. In addition, Lcarnitine suppressed atrogin-1 mRNA expression, which has been reported to play a pivotal role in muscle atrophy. The present study shows that L-carnitine has a protective effect against soleus muscle atrophy caused by hindlimb suspension and decreased E3 ligase mRNA expression, suggesting the possibility that L-carnitine protects against muscle atrophy, at least in part, through the inhibition of the ubiquitin proteasome pathway. These observations suggest that L-carnitine could serve as an effective supplement in the decrease of muscle atrophy caused by weightlessness, in the fields of clinical and rehabilitative research.

Keywords: weightless, catabolic condition, hindlimb suspension, muscle atrophy, L-carnitine, musclespecific ubiquitin E3 ligases 


\section{Introduction}

Skeletal muscle atrophy is commonly associated with a decrease in protein synthesis and an increase in protein degradation, or by simultaneous changes in both processes; it occurs in a variety of conditions leading to catabolism, such as prolonged periods of bed rest, immobilization, denervation, weightless (Herningtyas et al. 2008; Zhang et al. 2007). In particular, injuries that occur in the sports field, such as ligament injury, fracture, and cartilage damage, often necessitates prolonged inactivity or immobilization, which is accompanied with muscle atrophy. Characteristics of muscle atrophy include reduced muscle fiber size and weight and a general shift from slow twitch to fast twitch muscles, which lead to decreased muscle quality and fatigue resistance and can affect health and physical function (Cohen et al. 2009; Jackman and Kandarian 2004; Servais et al. 2007). Therefore, it is important to find a nutritional intervention for minimizing reduced muscle function during weightlessness, after musculoskeletal injury.

In muscle atrophy caused by catabolic conditions, such as cancer, diabetes, denervation, and disuse atrophy, muscle protein is degraded mainly by the ubiquitin proteasome pathway (UPP) (Jagoe et al. 2001; Taillandier et al. 1996). The essential feature of protein degradation via the UPP is that it involves 2 separate and consecutive steps: proteins are tagged with a polyubiquitin chain by covalent attachment, and this tagging marks them for degradation by the $26 \mathrm{~S}$ proteasome complex (Glickman and Chechanover 2002; Tisdale 2005). Conjugation of ubiquitin to the target protein is performed in 3 steps via a covalent attachment by involving the sequential actions of E1, E2, E3 enzymes. Especially in these enzymes E3 ubiquitin ligase catalyzes the covalent attachment of ubiquitin to the target protein in the last step of the conjugation process. This process is repeated and multiple ubiquitin molecules to the target protein linked polyubiquitin chain formation, and the target protein that is recognized by the $26 \mathrm{~S}$ proteasome complex and rapidly degraded into smaller peptides (Cao et al. 2005; Thrower et al. 2000). Atrogin-1 (muscle atrophy F-box, also called MAFbx) and MuRF 1 
(muscle ring finger 1), both of which are muscle-specific ubiquitin E3 ligases, have been shown to significantly increase muscle during atrophy. The functional importance of 2 genes was identified in multiple models of skeletal muscle atrophy. Mice lacking both atrogin-1 and MuRF1 show normal growth curves and skeletal muscle weight induced by denervation. Moreover, deletion of MuRF1 mice attenuates the dexamethasone induced muscle atrophy and knockdown of MAFbx mice reduces muscle atrophy during fasting. Therefore, these data suggest that both E3 ligases from the ubiquitin proteasome pathway in skeletal muscle atrophy can be considered as reliable markers (Bodine et al. 2001; Cohen et al. 2009; Gomes et al. 2001).

L-carnitine is mostly stored in the muscle and is an essential factor for normal tissue function. Its most well-documented function is the translocation of long-chain fatty acids from the cytosol into the mitochondrial matrix for subsequent ß-oxidation (Kerner and Hoppel 2000; McGarry and Brown 1997). Recently, L-carnitine administration was found to suppress the gene expression of ubiquitin proteasome pathway in growing pigs (Keller et al. 2012) and growing rats (Keller et al. 2013). Furthermore, it has been reported that L-carnitine down-regulates atrogin-1 and MuRF1 mRNA expression and reduces muscle wasting in a rat model of cancer cachexia (Busquetes et al. 2012). Lcarnitine administration can increase the level of insulin-like growth factor-1 (IGF-1) (Keller et al., 2013), which in turn activates the downstream PI3K/Akt/mTOR signaling pathway (Schiaffino and Mammucari 2011). Activation of $\mathrm{PI} 3 \mathrm{~K} / \mathrm{Akt} / \mathrm{mTOR}$ signaling increases protein synthesis and decreases muscle atrophy through the inhibition of muscle-specific E3 ligase transcription factors such as FoxO (Bodine et al. 2001; Boister et al. 2003; Glass 2003; Manning and Cantley 2007). Altogether, these findings suggest that L-carnitine administration may protect muscle from atrophy. However, the effect of L-carnitine administration in growing rats on the simultaneous change of morphological and degradation of both proteins and synthesis pathways in disuse induced muscle atrophy, such as hindlimb suspension, has not yet been studied.

We hypothesized that L-carnitine may have a protective effect on muscle atrophy induced by 
prolonged hindlimb suspension via the activation of Akt1/mTOR or inhibition of UPP. To examine this hypothesis, we selected hindlimb suspension induced muscle atrophy of growing rats as a model of weightless muscle atrophy.

\section{Materials and Methods}

\section{Animals}

Male Wistar rats were purchased from Orient Bio (Seongnam, Korea) at 6 weeks of age $(\mathrm{n}=21$; mean weight $=286 \mathrm{~g})$. All animals were housed in a climate controlled room in a $12 \mathrm{~h}$ light/dark cycle, and were fed rat chow and water ad libitum during the entire experimental period. All experimental procedures were carried out with the approval of the Institutional Animal Care and Use Committee of Konkuk University. The protocol was approved by the Committee on the Ethics of Animal Experiments of the Konkuk University (Permit number: KU11055-3).

\section{Experimental design}

After 1 week of acclimatization, rats were randomly assigned to a hindlimb suspension group ( $\mathrm{n}=$ 7), hindlimb suspension with L-carnitine administration group $(\mathrm{n}=7)$, and a pair-fed group $(\mathrm{n}=7)$. Because it is known that food intake decreases during hindlimb suspension (Morey-Holton and Globus, 2002), the pair-fed group received the same amount of food as that consumed by both hindlimb suspension groups during the previous week. The L-carnitine group were administered a $1250 \mathrm{mg}$ L-carnitine/kg (purchased from Sigma-Aldrich, St Louis, USA) dissolved in distilled water orally using a sonde (Keller et al., 2013). The body weight was measured every morning at 09:00 and L-carnitine solution was ingested every morning at 10:00. The experiment was conducted for 14 days. 


\section{Hindlimb suspension}

The procedure of hindlimb suspension was performed according to the non-invasive method described by Morey-Holton and Globus (2002). Briefly, the tail of each rat was cleaned with 70\% ethanol and then sprayed with a generous amount of adhesive to increase the adhesive forth (Mueller Sports Medicine Inc., Germany). After this, the tail was wrapped with an elastic tape (5cm width) and suspended at the top of the cage. Each rat was allowed $360^{\circ}$ rotation and was permitted to move freely through the use of its forelimbs for access to food and water. The rats were suspended at a $\sim 30^{\circ}$ angle head-down tilt to avoid contact between the hindlimbs and the ground.

\section{Sample collection}

After 14 days of hindlimb suspension, rats were killed under avertin-induced anesthesia. For analysis, soleus and extensor digitorum longus (EDL) muscle samples were excised. All muscle samples from both the left and right hindlimb were weighed. The muscles from the left side were immediately frozen in liquid nitrogen; those from the right side were covered with O.C.T. compound and quickly frozen in liquid nitrogen-cooled isopentane for immunohistochemistry analysis. All muscle samples were then stored at $-80^{\circ} \mathrm{C}$ until used.

\section{Immunohistochemistry}

For analysis of fiber type and size, muscle specimens from the right soleus of each rat were rapidly frozen in liquid nitrogen-cooled isopentane and sectioned to a thickness of $10 \mu \mathrm{m}$ on a cryostat at $20^{\circ} \mathrm{C}$. The muscle sections were air-dried and then washed with PBS, and placed in $0.3 \% \mathrm{H}_{2} \mathrm{O}_{2}$ for 10 min. After blocking with $1 \%$ skim milk, the samples were then incubated with diluted mouse antimyosin fast skeletal antibody (MY-32) (1:500, Novus, CO, USA) overnight at $4^{\circ} \mathrm{C}$ and subsequently exposed to biotinylated goat anti-mouse $\operatorname{IgG}$ and mouse anti-rabbit secondary antibodies (1:200, 
Vector, Burlingame, CA, USA). They were then visualized by reaction with 3,3'-diaminobenzidine tetrachloride (Sigma, St. Louis, MO, USA). Sections were mounted in Canada balsam (Kanto, Tokyo, Japan) following dehydration. The distribution of each fiber type has been expressed as a ratio between the number of fibers of each type and the total number of fibers. The size of at least 200 fibers from each rat muscle was measured with INFINITY lite software (Innerview 2.0, Lumenera, Canada).

\section{Total RNA extraction and reverse transcription}

Total RNA from soleus muscles was extracted using Trizol reagent (Life Technologies Inc.) in accordance with the manufacturer's protocol. Samples were treated with DNase, chloroform extract, and re-suspended in $20 \mu \mathrm{l}$ RNase-free water. cDNA was synthesized from $1 \mu \mathrm{g}$ of the total RNA using cDNA Synthesis Master Mix (GenDEPOT, CA, USA). Subsequently, RT-PCR was performed using amfico Taq DNA polymerase (GenDEPOT, CA, USA). The primer sequences are shown in Table 1.

The position of Table 1

\section{Western blotting}

Soleus muscle was homogenized using homogenization buffer and homogenizer. Homogenates were centrifuged at $4{ }^{\circ} \mathrm{C}$ and $12,000 \mathrm{rpm}$ for $15 \mathrm{mins}$, and then total protein concentration of the supernatants was determined using Bradford method. The samples were boiled at $95^{\circ} \mathrm{C}$ for 5 minutes. For each sample, a total protein concentration of $30 \mu \mathrm{g}$ was resolved by SDS-PAGE, using $6 \%$ (mTOR and p-mTOR), 12\% (Akt1 and p-Akt1) gels, and transferred to polyvinylidene fluoride (PVDF) membranes at 100 volt for $2 \mathrm{~h}$. After the membrane was blocked for $1 \mathrm{~h}$ with 5\% skimmed milk, the primary antibodies, including Akt (\#9272), p-AKT (Ser473) (\#4508), mTOR (\#2972), pmTOR (Ser2448) (\#29671, Cell signaling technology, MA, USA, dilution 1:1000) were incubated in 
a $5 \%$ BSA solution during overnight at $4^{\circ} \mathrm{C}$. After overnight incubation at $4^{\circ} \mathrm{C}$, the membrane washed with TBS-T and incubated with a donkey anti-rabbit IgG H\&L (HRP) (ab6802, Abcam, Cambridge, UK, dilution 1:1000) at room temperature for $1 \mathrm{~h}$. Immunodetection was carried out with ECL detection reagent (GE healthcare, Buckingham, UK). The protein amount analysis was performed using Image J software (National Institutes of Health).

\section{Data analysis}

All data are presented as the mean \pm SE. All statistical analyses were performed with SPSS version 19.0 software (SPSS, Inc., Chicago, IL, USA). One-way repeated ANOVA was used to determine overall differences. A LSD post hoc test was used to determine group differences. A p-value of $<0.05$ denoted statistical significance.

\section{Results}

\section{Food intake and body weight}

Since the pair-fed group's condition was maintained for 14 days in order to eliminate interference due to dietary differences, food consumption between this group and both hindlimb suspension groups did not differ (Fig. 1A). However, there were significant differences in the mean body weight after hindlimb suspension between the control group and both hindlimb suspension groups. The control group showed increased body weight during the 14 days, but both hindlimb suspension groups displayed slight increase after hindlimb suspension (Fig. 1B).

The position of Figure 1

\section{Soleus and EDL muscle weight}

During hindlimb suspension, the soleus muscle weight reduced in both hindlimb suspension and 
hindlimb suspension with L-carnitine administration groups, compared to controls (48\% and 37\%, respectively). However, the L-carnitine administration group tended to be $21 \%$ higher than the hindlimb suspension group in soleus muscle weight to body weight ratio, though this was not statistically significant $(\mathrm{p}=0.059)$ (Fig. 2A). The EDL muscle weight to body weight ratio did not differ between groups (Fig. 2B).

The position of Figure 2

\section{Muscle fiber size}

Immunohistochemistry staining of fast myosin heavy chain (MY-32) was performed on soleus muscle fibers for a representative control and hindlimb suspension groups, with and without Lcarnitine at 14 days after hindlimb suspension (Fig. 3A). After hindlimb suspension, soleus muscle fiber size significantly reduced in hindlimb suspension and hindlimb suspension with L-carnitine administration groups, compared to the control group (46\% and 34\%, respectively). However, the Lcarnitine significantly attenuated total and type I and type II muscle fiber size decrease in soleus muscle (Fig. 3B, C and D).

\section{The position of Figure 3}

\section{Distribution of muscle fiber size}

There were differences in the size distribution of total and individual soleus muscle fiber types between hindlimb suspension and hindlimb suspension with L-carnitine administration groups. In the hindlimb suspension group, the distribution of total and type I and II fiber size shifted left (i.e., to a smaller size), compared to the hindlimb suspension with L-carnitine administration group (Figs. 4A, B and C).

The position of Figure 4

\section{Atrogin-1 and MuRF1 mRNA levels induced by hindlimb suspension}


To elucidate whether the muscle specific E3 ubiquitin ligases are inhibited by L-carnitine on hindlimb suspension-induced muscle atrophy, we measured atrogin-1 and MuRF1 mRNA expression in soleus muscles. L-carnitine significantly attenuated the atrogin-1 mRNA expression compared to that noted in the hindlimb suspension group (Figs. 5A) but did not significantly attenuate the MuRF1 mRNA expression (Fig. 5B) in the soleus muscle $(\mathrm{p}=0.074)$.

The position of Figure 5

\section{Akt1 and mTOR mRNA levels induced by hindlimb suspension}

To determine whether Akt1/mTOR pathway is affected by L-carnitine, we measured the Akt1 and mTOR mRNA levels in soleus muscles. After hindlimb suspension for 14 days, these were significantly reduced compared to the control group, but there was no difference between both hindlimb suspension groups (Fig. 6 A and B)

The position of Figure 6

\section{phosphorylated mTOR protein level induced by hindlimb suspension}

To determine whether Akt1/mTOR pathway is affected by L-carnitine, we measured the ratios of phospho-Akt1 to total Akt1 and phospho-mTOR to total mTOR protein levels in soleus muscles. After hindlimb suspension for 14 days, the ratios of phospho-mTOR (Ser2448) to total mTOR protein levels significantly reduced in hindlimb suspension group compared to the control group but not L-carnitine group. However, the L-carnitine administration group tended to higher than the hindlimb suspension group, though this was not statistically significant (Fig. 7 A). And we also measured the ratio of phospho-Akt1 to total Akt1 protein levels, but phosphorylation of Akt1 (Ser473) was not detected in all groups.

The position of Figure 7

\section{Discussion}

Morey-Holton and Globus (2002) reported that food consumption may decrease and then increase 
for a few days during the experiment, and that body weight would stabilize or slightly increase throughout the 14 days of hindlimb suspension (Ishihara et al. 2004). In this study, body weight gain in both groups slightly increased for the 14 days of hindlimb suspension and food intake decreased for the first 2 days, showing a tendency to increase again after the first 3 days. Our findings are consistent with previous research, which showed that, in growing rats without a catabolic condition, L-carnitine administration did not influence food intake or body weight gain in growing rats (Keller et al. 2013).

In the present study, hindlimb suspension reduced the weight of the soleus muscle but not the EDL muscle, in both hindlimb suspension groups. However, soleus muscle weight loss was not due to body weight loss, because muscle weight loss occurred in only the soleus muscle and not the EDL muscle. It has been well established that compared to the EDL muscle, the soleus muscle shows more pronounced atrophy when the individual is subjected to hindlimb suspension; this is because hindlimb suspension primarily affects slow-twitch and anti-gravity muscles such as the soleus muscle (Desplanches et al. 1987, Fitts et al. 2000, Hurst and Fitts 2003), which is consistent with our results. Although these results are consistent with our findings, administration of L-carnitine still reduced the soleus muscle weight during hindlimb suspension compared to the hindlimb suspension group lacking L-carnitine.

To the best of our knowledge, this is the first study investigating the morphological adaptation of soleus muscle after hindlimb suspension corresponding to L-carnitine administration in growing rats. We found that L-carnitine administration leads to the prevention of decrease in muscle fiber size during hindlimb suspension. Moreover, the L-carnitine administration group showed a tendency to retain a larger fiber size compared with the hindlimb suspension group for total, type I, and type II fibers. This finding agrees with a recent study using other nutritional interventions such as BCAAs and vitamin E. Servais et al. (2007) showed that hindlimb suspension led to a decrease in the size of type I $(\sim 59 \%)$ and II ( $42 \%)$ fibers, and vitamin E supplementation decreased the rate of muscle 
proteolysis by reducing the expression of calpains, caspases-3, -9 , and -12 , and $\mathrm{E}_{3}$ ubiquitin ligases (MuRF1 and MAFbx). Furthermore, Maki et al. (2012) reported that BCAA administration partly prevented $(15 \%)$ the decrease in soleus muscle fiber size compared to the control group, during 14 days of hindlimb suspension. Therefore, L-carnitine appears to have the potential to prevent muscle atrophy in rats during hindlimb suspension, similar to other nutritional interventions such as BCAA and vitamin E.

The ubiquitin proteasome pathway (UPP), known as a primary pathway, is associated with disuse muscle atrophy (Jagoe and Goldberg 2001; Lecker et al. 2004; Price 2003). In the muscle, UPP is controlled by 2 muscle-specific ubiquitin E3 ligases, such as atrogin-1 and MuRF1, both of which are involved in protein degradation in muscle and are increased in muscle atrophy (Bodine et al. 2001; Gomes et al. 2001; Lecker et al. 2004). The induction of two muscle-specific E3 ligases mRNA expression is significantly increased in early phase during hindlimb suspension. However, the activation was transient, fading away after 7 days of hindlimb suspension (Cannavino et al. 2014). In this study, after 14 days of hindlimb suspension, there were significant MuRF1 down-regulation in both hindlimb suspension groups, whereas atrogin-1 mRNA expression was lower in only L-carnitine administration group compared to control group. Although, we did not observe the change of E3 ligases in early phase of hindlimb suspension, this results similar to previous study. And, recent studies have shown that L-carnitine administration reduced the muscle-specific E3 ligase expression (atrogin-1 and MuRF1) in the skeletal muscle (Busquetes et al. 2012; Keller et al. 2012; Keller et al. 2013). In this study, we found that L-carnitine administration significantly suppressed the expression of the muscle-specific E3 ligases, especially atrogin-1 mRNA expression in soleus muscle, compared to the hindlimb suspension group. This result is in agreement with recent studies. Keller et al. (2012) showed that L-carnitine administration reduced atrogin-1 and MuRF1 mRNA levels in the skeletal muscle of growing pigs, and Busquetes et al. (2012) reported that L-carnitine decreased MuRF1 mRNA expression in the gastrocnemius muscle of rats suffering from cancer cachexia. Although the 
underlying mechanism by which L-carnitine decrease muscle-specific E3 ligases levels is not fully understand, L-carnitine successfully decreases weightless muscle atrophy induced by hindlimb suspension, at least in part, through the inhibition of E3 ligase mRNA expression, especially that of atrogin-1.

Furthermore, recent studies confirmed that L-carnitine administration increases plasma IGF-1 concentration in chicks (Kita et al. 2002), pigs (Doberenz et al. 2006), and rats (Heo et al. 2001). Increase in IGF-1 activates a protein synthesis pathway, called the Akt/mTOR signaling pathway. The binding of IGF-1 to its receptor triggers the activation of this downstream signaling pathway. The Akt/mTOR pathway is up-regulated during hypertrophy and down-regulated during muscle atrophy (Bodine et al. 2001). Furthermore, the IGF-1/Akt/mTOR pathway decreases muscle atrophy via the inhibition of the muscle-specific E3 ligase transcription factor family, known as the forkhead box-O transcription factors (FoxOs) (Boister et al. 2003; Glass et al. 2003; Manning et al. 2007; Stitt et al. 2004). Thus, we examined whether Akt/mTOR pathway is affected by L-carnitine during hindlimb suspension in mRNA and protein levels. In this study, Akt1 and mTOR mRNA expression significantly reduced in both hindlimb suspension groups, but did not L-carnitine attenuate the decrease of Akt1 and mTOR mRNA level compared to the hindlimb suspension group. And Lcarnitine tended to higher than the hindlimb suspension group in phospho-mTOR (Ser2448) to total mTOR protein level, though this was not statistically significant. Also, we measured the phosphoAkt1 to total Akt1 protein level, but phosphorylation of Akt1 (Ser473) was not detected in all groups. We could not find a link between L-carnitine and Akt/mTOR pathway. In contrast to our result, Keller et al. (2013) reported that L-carnitine increased the phosphorylated Akt1 and mTOR protein levels in the quadriceps femoris muscle of growing rats without a catabolic condition. This difference in results on the Akt1/mTOR pathway between our study and Keller et al. study may be due to differences in the protein catabolic conditions and the measured muscle.

\section{Conclusion}


In conclusion, this study provides evidence for the significant effects of L-carnitine administration on the prevention of muscle atrophy through the suppression of UPP without the activation of Akt1/mTOR signaling pathway during weightlessness in growing rats. However, we did not investigate the dose-response of L-carnitine. Further studies are needed to examine dose-response relationships and assess whether similar effects can also be observed in humans. Our data shows that L-carnitine has a protective effect against soleus muscle atrophy caused by hindlimb suspension and it decreased E3 ligase mRNA expression without activating Akt/mTOR pathway, suggesting the possibility that L-carnitine protects against muscle atrophy, at least in part, through the inhibition of the UPP. These observations suggest that L-carnitine could serve as an effective supplement in the prevention or treatment of muscle atrophy caused by weightlessness in the fields of clinical and rehabilitative research.

\section{Conflict of interest statement}

The authors declare that there are no conflicts of interest.

\section{Acknowledgements}

We would like to express thanks to all the participants for their engagement in this study. 


\section{References}

Baehr, L.M., Furlow J.D., and Bodine S.C. 2011. Muscle sparing in muscle RING finger 1 null mice: response to synthetic glucocorticoids. The Journal of physiology. 589(19): 4759-4776.

Bodine, S.C., Stitt, T.N., Gonzalez, M., Kline, W.O., Stover, G.L., Bauerlen, R., et al. 2001. Akt/mTOR pathway is a crucial regulator of skeletal muscle hypertrophy and can prevent muscle atrophy in vivo. Nature Cell Biology. 3: 1014-1019.

Boister, D.R., Kubica, N., Crozier, S.J., Williamson, D.L., Farrell, P.A., Kimball, S.R., et al. 2003. Immediate response of mammalian target of rapamycin (mTOR)-mediated signalling following acute resistance exercise in rat skeletal muscle. The Journal of Physiology. 554(1): 213-220.

Busquetes, S., Serpe, R., Toledo, M., Betancourt, A., Marmonti, E., Orpi, M., et al. 2012. 1-Carnitine: An adequate supplement for a multi targeted anti wasting therapy in cancer. Clinical Nutrition. 31: 889-895.

Cannivino, J., Brocca, L., Sandri, M., Bottinelli, R., Pellegrino, M.A. 2014. PGC-1 $\alpha$ over-expression prevents metabolic alterations and soleus muscle atrophy in hindlimb unloaded mice. J Physiol. 592(20): 4575-4589.

Cao, P.R., Kim, H.J., Lecker, S.H. 2005. Ubiquitin-protein ligases in muscle wasting. The International Journal of Biochemistry \& Cell Biology. 37: 2088-2097.

Clavel, S., Coldefy, A.S., Kurkdjian, E., Salles, J., Margaritis, I., Derijard, B. 2006. Atrophy-related 
ubiquitin ligases, atrogin-1 and MuRF1 are up regulated in aged rat tibialis anterior muscle. Mechanism of Ageing and Development. 127: 794-801.

Cohen, S., Brault, J.J., Gygi, S.P., Glass, D.J., Valenzuela, D.M., Gartner, C., et al. 2009. During muscle atrophy, thick but not thin, filament components are degraded by MuRF1-dependent ubiquitylation. Journal of Cell Biology. 185: 1083-1095.

Cong, H., Sun, L., Liu, C., and Tien, P. 2011. Inhibition of atrogin-1/MAFbx expression by adenovirus-delivered small hairpin RNAs attenuates muscle atrophy in fasting mice. Hum Gen Ther. 22: 1-12.

Desplanches, D., Mayet, M.H., Sempore, B., Flandrois, R. 1987. Structural and functional responses to prolonged hindlimb suspension in rat muscle. J Appl Physiol. 63: 558-63.

Doberenz, J., Birkenfeld, C., Kluge, H., Eder, K. 2006. Effects of L-carnitine supplementation in pregnant sows on plasma concentrations of insulin-like growth factors, various hormones and metabolites and chorion characteristics. J Anim Physiol Nutr. 90: 487-499.

Fitts, RH., Riley, D.A., Widrick, J.J. 2000. Physiology of a micro-gravity environment invited review: microgravity and skeletal muscle. J Appl Physiol. 89: 823-839.

Glass, D.J. 2003. Signaling pathway that mediate skeletal muscle hypertrophy and atrophy. Nature Cell Biology. 5: 87-90.

Glickman, M.H., Ciechanover, A. 2002. The Ubiquitin-Proteasome Proteolytic Pathways: 
Destruction for the Sake of Construction. American Journal of Society. 82: 373-428.

Gomes, M.D., Lecker, S.H., Jagoe, R.T., Goldberg, A.L. 2001. Atrogin-1, muscle-specific F-box protein highly expressed during muscle atrophy. Proceeding of the National Academic Sciences. 98: 1440-1445.

Haddad, F., Adams, G.R., Bodell, P.W., Baldwin, K.M. 2006. Isometric resistance exercise fails to counteract skeletal muscle atrophy processes during the initial stages of unloading. J Appl Physiol. 100: $433-441$.

Heo, Y.R., Kang, C.W., Cha, Y.S. 2001. L-carnitine changes the levels of insulin-like growth factors(IGFs) and IGF binding proteins in streptozotocin-induced diabetic rat. J Nutr Sci Vitaminol. 47: $329-334$.

Herningtyas, E.H., Okimura, Y., Handayaningsih, A.E., Yamamoto, D., Maki, T., Iida, K., et al. 2008. Branched-chain amino acids and arginine suppress MaFbx/atrogin-1 mRNA expression via mTOR pathway in C2C12 cell line. Biochemistry et Biophysian Acta. 1780: 1115-1120.

Hurst, J.E., Fitts, R.H. 2003. Hindlimb unloading-induced muscle atrophy and loss of function: protective effect of isometric exercise. J Appl Physiol. 95: 1405-1417.

Ishihara, A., Kawano, F., Ishioka, N., Oishi, H., Higashibata, A., Shimazu, T., et al. 2004. Effects of running exercise during recovery from hindlimb unloading on soleus muscle fibers and their spinal motoneurons in rats. Neurosci Res. 48: 119-127. 
Jackman, R.W., Kandarian, S.C. 2004. The molecular basis of skeletal muscle atrophy. American Journal of Physiology. 284: 834-843.

Jagoe, R.T., Goldberg, A.L. 2001. What do we really know about the ubiquitin-proteasome pathway in muscle atrophy? Curr Opin Clin Nutr Metab Care. 4: 183-190.

Keller, J., Ringseis, R., Koc, A., Luckas, I., Kluge, H., Eder, K. 2012. Supplementation with Lcarnitine downregulates genes of the ubiquitin proteasome pathway in the skeletal muscle and liver of piglets. Animal. 6: 70-78.

Keller, J., Couturier, A., Haferkamp, M., Most, E., Eder, K. 2013. Supplementation of carnitine leads to an activation of the IGF-1/PI3K/Akt signaling pathway and down regulates the E3 ligase MuRF1 in skeletal muscle of rats. Nutrition and Metabolism. 10: 28.

Kerner, J., Hoppel, C. 2000. Fatty acid import into mitochondria. Biochmistry et Biophysica Acta. 1486: $1-17$.

Kita, K., Kato, S., Amanyaman, M., Okumura, J., Yokota, H. 2002. Dietary L-carnitine increases plasma insulin-like growth factor-1 concentration in chicks fed a diet with adequate dietary protein level. Br Poult Sci. 43: 117-121.

Lecker, S.H., Jagoe, R.T., Gilbert, A., Gomes, A., Gomes, M., Baracos, V., et al. 2004. Multiple types of skeletal muscle atrophy involve a common program of changes in gene expression. FASEB.

18: $39-51$ 
Maki, T., Yamamoto, D., Nakanishi, S., Iida, K., Iguchi, G., Takahashi, Y., et al. 2012. Branchedchain amino acids reduce hindlimb suspension-induced muscle atrophy and protein levels of atrogin1 and MuRF1 in rats. Nutrition Research. 32: 676-683.

Manning, B.D., Cantley, L.C. 2007. Akt/PKB signaling: Navigating Downstream. Cell. 129: 12611274.

McGarry, J.D., Brown, N.F. 1997. The mitochondrial carnitine palmitoyltransferase system from concept to molecular analysis. European Journal of Biochemistry. 244: 1-14.

Morey-Holton, E.R., Globus, R.K. 2002. Hindlimb unloading rodent model: technical aspects. J Appl Physiol. 92: 1367-1377.

Oishi, Y., Ogata, T., Yamamoto, K., Terada, M., Ohira, T., Ohira, Y., et al. 2008. Cellular adaptation in soleus muscle during recovery after hindlimb unloading. Acta Pshysiol. 192: 381-395.

Price, S.R. 2003. Increased transcription of ubiquitin-proteasome system components: molecular responses associated with muscle atrophy. IJBCB. 35: 617-628

Schiaffino, S., Mammucari, C. 2011. Regulation of skeletal muscle growth by the IGF1-Akt/PKB pathway: insights from genetic models. Skeletal muscle. 1(1): 4.

Servais, S., Letexier, D., Favier, R., Duchamp, C., Desplanches, D. 2007. Prevention of unloadinginduced atrophy by vitamin E supplementation: Links between oxidative stress and soleus muscle proteolysis? Free Radical Biology \& Medicine. 42: 627-635. 
Stitt, T.N., Brian, D.D., Clarke, B.A., Panaro, F., Tomofeyva, Y., Kline, W.O., et al. 2004. The IGF1.PI3K/Akt pathway prevents expression of muscle atrophy-induced ubiquitin ligases by inhibiting FOXO transcription factor. Molecular Cell. 14: 395-403.

Taillandier, D., Aurousseau, E., Meynial-Denis, D., Bechet, D., Ferrara, M., Cottin, P., et al. 1996. Coordinate activation of lysosomal, $\mathrm{Ca}^{2+}$-activated and ATP-ubiquitin-dependent proteinases in the unweighted rat soleus muscle. Biochem J. 316: 65-72.

Thrower, J.S., Hoffman, L., Rechsteiner, M., Pickart, C.M. 2000. Recognition of the polyubiquitin proteolytic signal. European Molecular Biology Organization. 19: 94-102.

Thomason, D.B., Booth, F.W. 1990. Atrophy of the soleus muscle by hindlimb unweighting. J Appl Physiol. 68: 1-12.

Tisdale, M.J. 2005. The Ubiquitin-Proteasome Pathway as a Therapeutic Target for Muscle Wasting. J support Oncol. 3(3): 209-217.

Zhang, H., He, Z., Gao, Y., Hinghofer-Szalkay, H., Fan, X. 2007. Muscle Composition After 14-Day Hindlimb Unloading in Rats: Effects of Two Herbal Compounds. Aviation, Space, and Environmental Medicine. 78(10): 926-931. 
Table 1.

Table 1. Primer sequences for RT-PCR

\begin{tabular}{|c|c|c|}
\hline Target mRNA & Forward primer & Reverse primer \\
\hline Atrogin-1 & 5' GAC TGG ACT TCT CGA CTG CC 3' & 5' GAC TTG CCG ACT CTC TGG AC 3' \\
\hline MuRF1 & 5' ACA TCT TCC AGG CTG CCA AT 3' & 5' GTT CTC CAC CAG CAG GTT CC 3' \\
\hline Akt1 & 5' TGC TGG AGG ACA ACG ACT AT 3' & 5' TGT CAT CTT GAT CAG GCG GT 3' \\
\hline mTOR & 5' TTG AGG TTG CTA TGA CCA GAG AGA A 3' & 5' TTA CCA GAA AGG ACA CCA GCC AAT G 3' \\
\hline GAPDH & $5^{\prime}$ TGC TGG TGC TGA GTA TGT CG 3' & $5^{\prime}$ TGA TGG CAT GGA CTG TGG TC $3^{\prime}$ \\
\hline
\end{tabular}




\section{Figure Captions}

Figure 1. Effects of L-carnitine on food intake and body weight. (A) Food intake. (B) Body weight. Date are presented as mean \pm SE. $\mathrm{n}=7$ each group. $*$ Significantly differ from control group $(P<0.05)$. Control, pair-fed; HS, hindlimb suspension; HS + Carnitine, hindlimb suspension with Lcarnitine.

Figure 2. Effects of L-carnitine on the soleus and EDL muscle weight. (A) Ratio of soleus muscle weight to body weight after hindlimb suspension. (B) Ratio of EDL muscle weight to body weight after hindlimb suspension. Date are presented as mean \pm SE. $n=7$ each group. ${ }^{*}$ Significantly differ from control group $(P<0.05)$. Control, pair-fed; HS, hindlimb suspension; HS + Carnitine, hindlimb suspension with L-carnitine.

Figure 3. Immunohistochemistry staining of fast myosin heavy chain (MY-32) in soleus muscle fibers. (A) Type I muscle fiber is seen as unstained area (white), and type II muscle fiber as stained area (brown). Scale bars represent $100 \mu \mathrm{m}$. (B), (C) and (D), Mean size of total, type I, and type II fibers of the soleus muscle after hindlimb suspension. Date are presented as mean \pm SE. $n=5$ each group. * Significantly differ from control group $(P<0.05)$. $\dagger$ Significantly different from HS $(P<0.05)$. Control, pair-fed; HS, hindlimb suspension; HS + Carnitine, hindlimb suspension with L-carnitine.

Figure 4. Distribution of fiber size of the soleus muscle. $x$ axis is fiber size, sectioned by $300 \mu \mathrm{m}^{2}$; y axis indicates the percentage to total fiber numbers. (A), (B), and (C) indicate the distribution of total, type I, and type II fiber size. $\mathrm{n}=5$ each group. HS, hindlimb suspension; HS + Carnitine, hindlimb suspension with L-carnitine. 
Figure 5. Atrogin-1 and MuRF1 mRNA levels in the soleus muscle after 14-day hindlimb suspension. (A) Ratio of Atrogin-1 mRNA level to GAPDH after hindlimb suspension. (B) Ratio of MuRF1 mRNA level to GAPDH after hindlimb suspension. Date are presented as mean \pm SE. $n=7$ each group. * Significantly differ from control group $(P<0.05)$. $\dagger$ Significantly different from HS $(P<0.05)$. Control, pair-fed; HS, hindlimb suspension; HS + Carnitine, hindlimb suspension with Lcarnitine.

Figure 6. Akt1 and mTOR mRNA levels in the soleus muscle after 14-day hindlimb suspension. (A) Ratio of Akt1 mRNA level to GAPDH after hindlimb suspension. (B) Ratio of mTOR mRNA level to GAPDH after hindlimb suspension. Date are presented as mean \pm SE. $\mathrm{n}=7$ each group. * Significantly differ from control group $(P<0.05)$. Control, pair-fed; HS, hindlimb suspension; HS + Carnitine, hindlimb suspension with L-carnitine.

\section{Figure 7. phosphorelated mTOR protein levels in the soleus muscle after 14-day hindlimb} suspension. (A) Ratio of phospho-mTOR protein level to mTOR after hindlimb suspension. Date are presented as mean \pm SE. $\mathrm{n}=7$ each group. ${ }^{*}$ Significantly differ from control group $(P<0.05)$. Control, pair-fed; HS, hindlimb suspension; HS + Carnitine, hindlimb suspension with L-carnitine. 
Figure 1

A

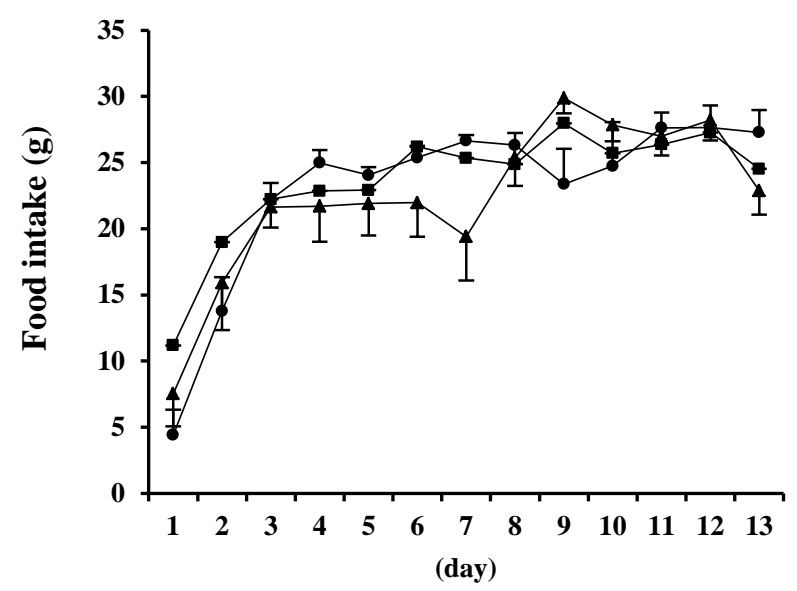

$\longrightarrow$ Control $\longrightarrow$ HS $\backsim$ HS+Carnitine
B

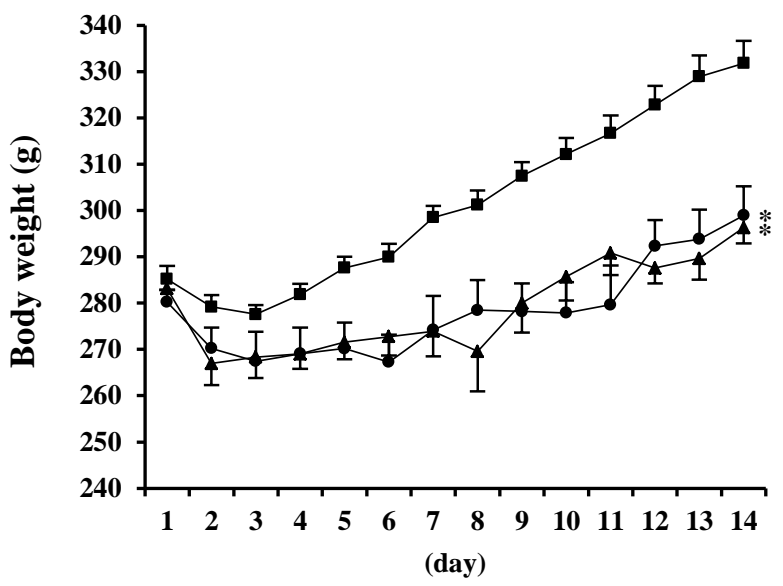

Control $\longrightarrow$ HS $\multimap$ HS+Carnitine 
Figure 2

A Soleus

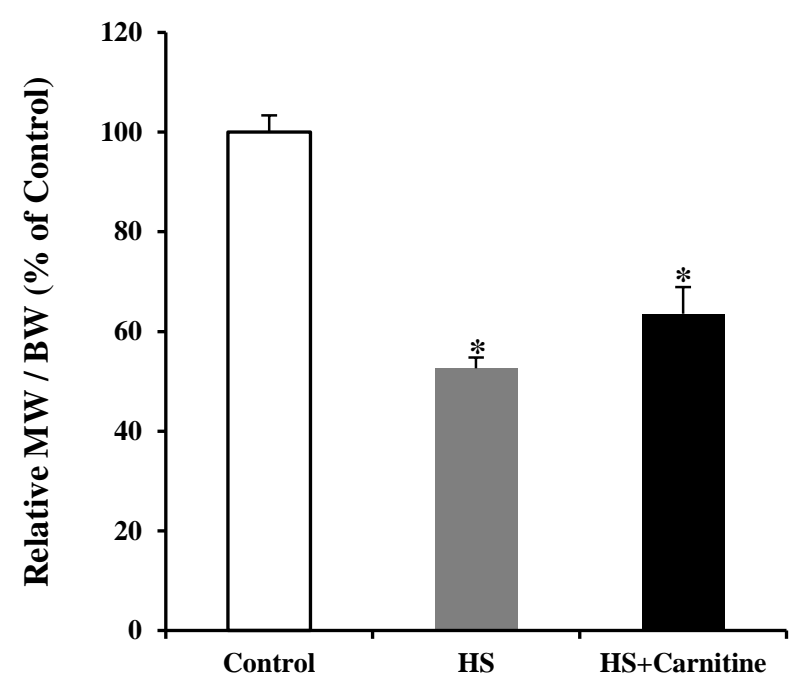

B EDL

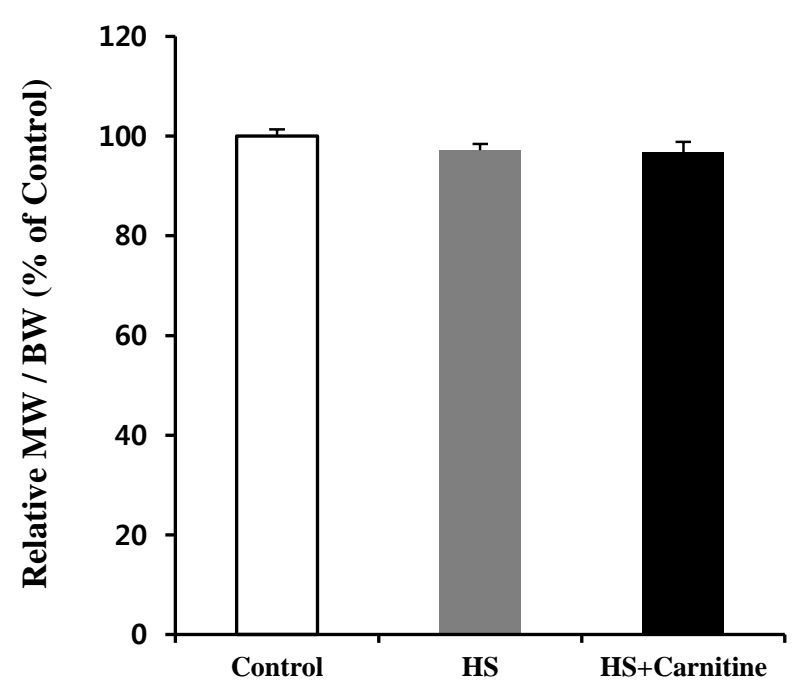


Figure 3

A

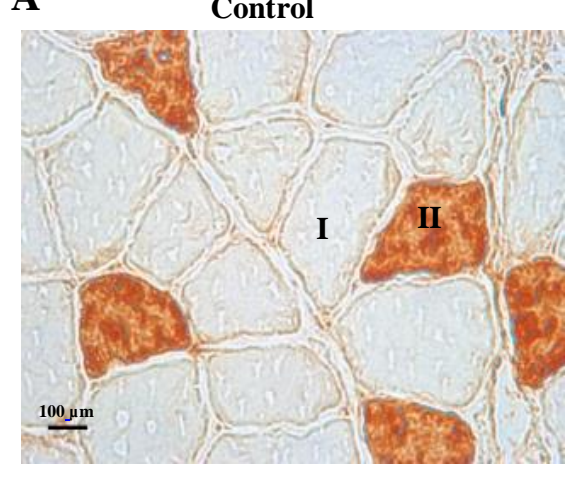

B

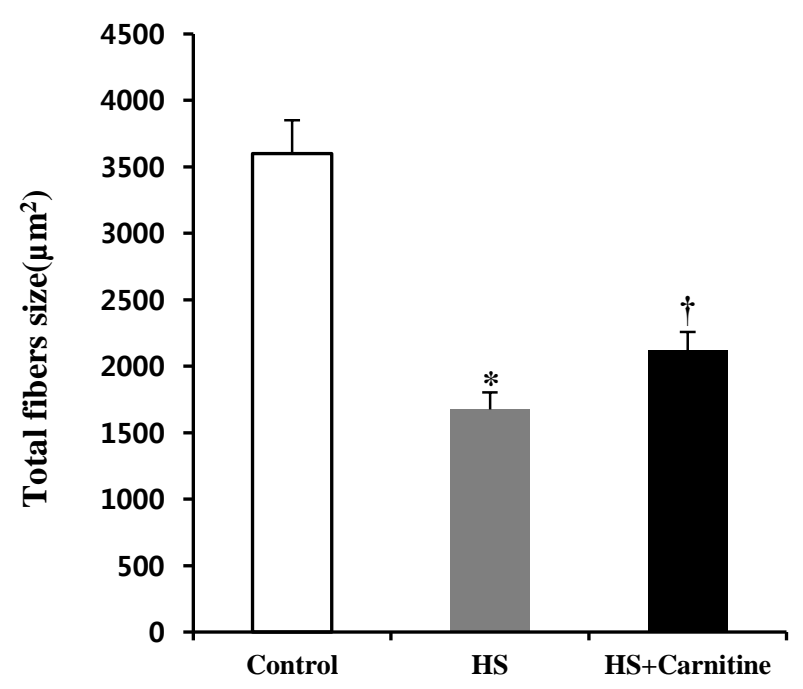

HS

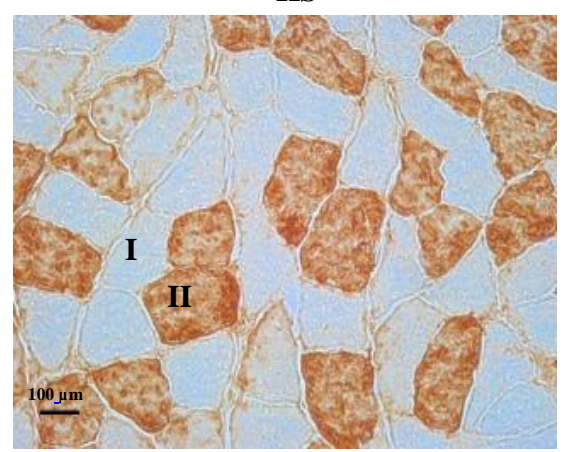

HS+carnitine

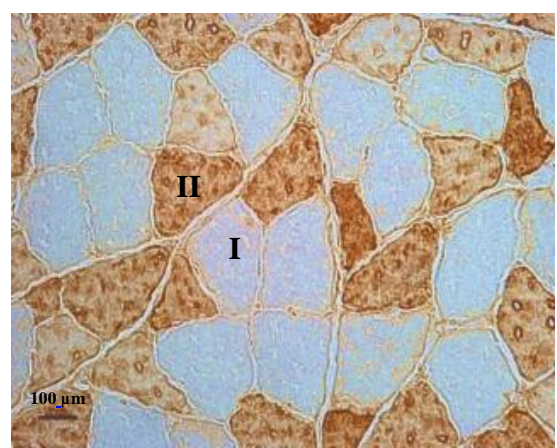

C

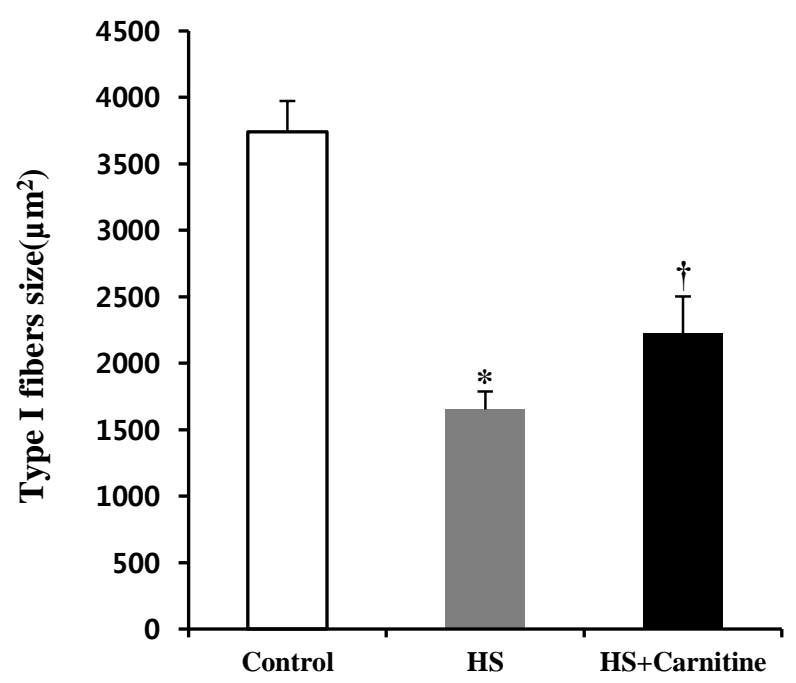

D

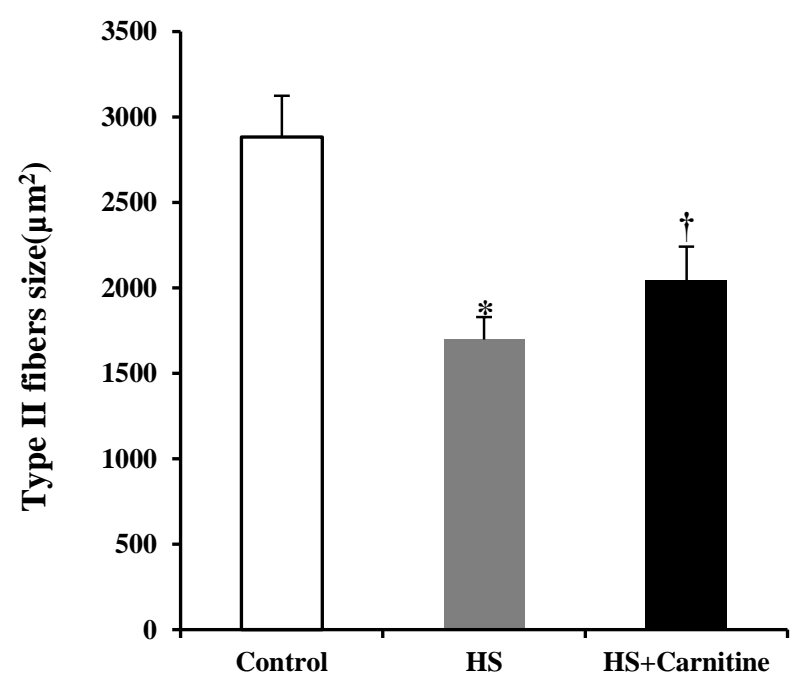


Figure 4

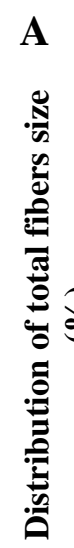

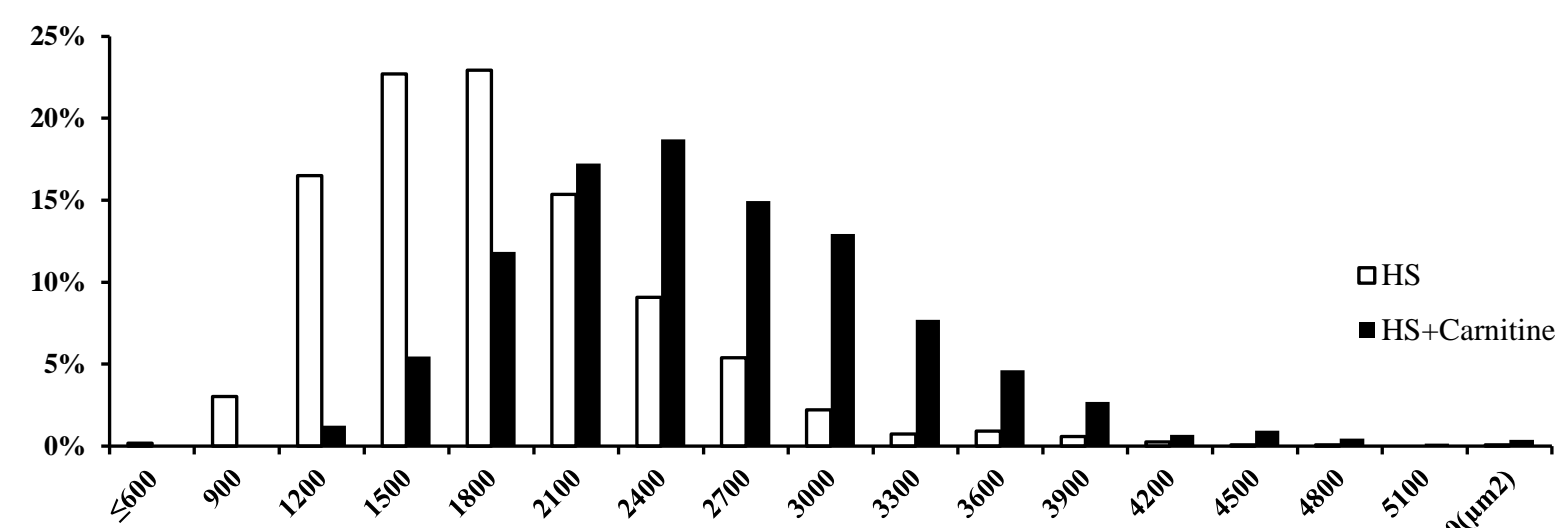

B

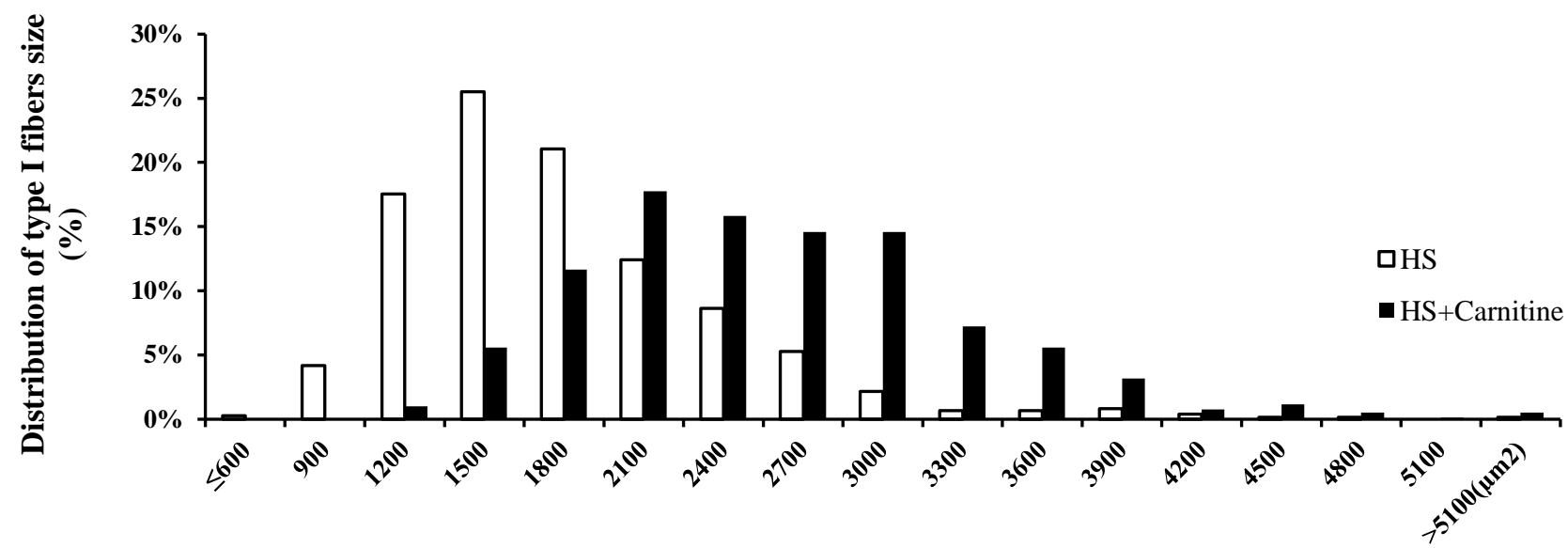

C

范

बे

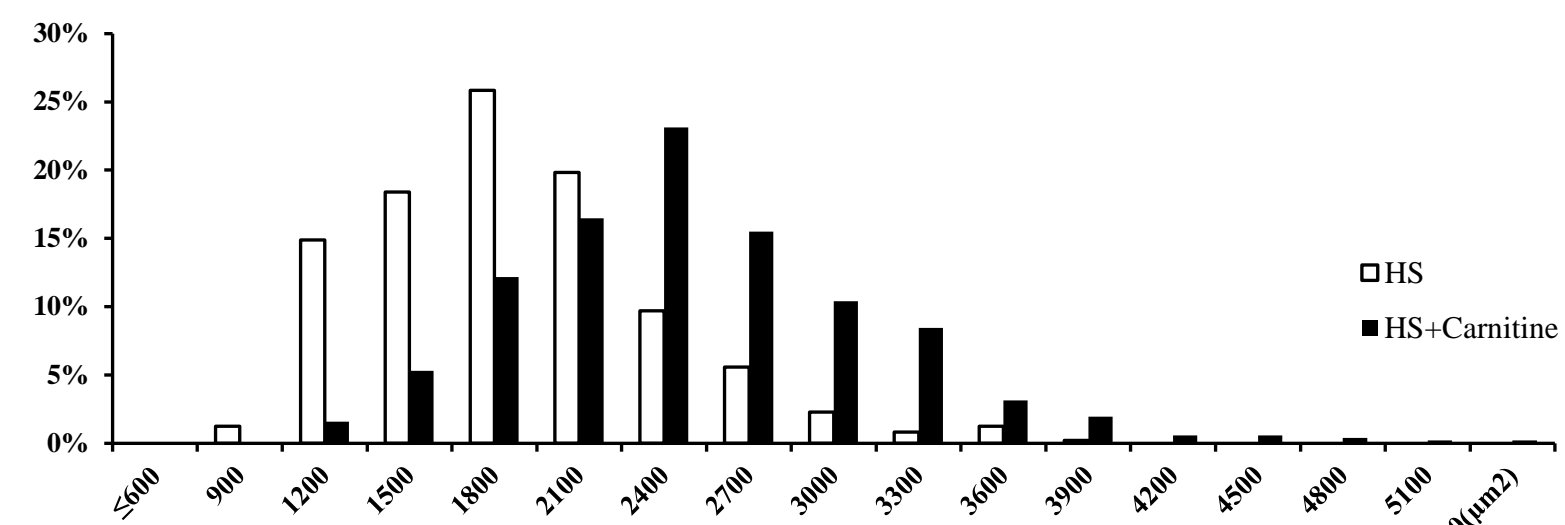


Figure 5
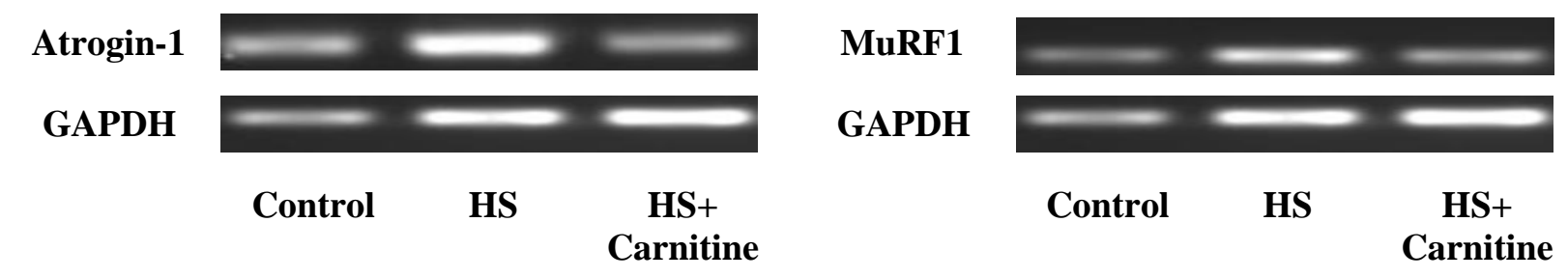

A

B
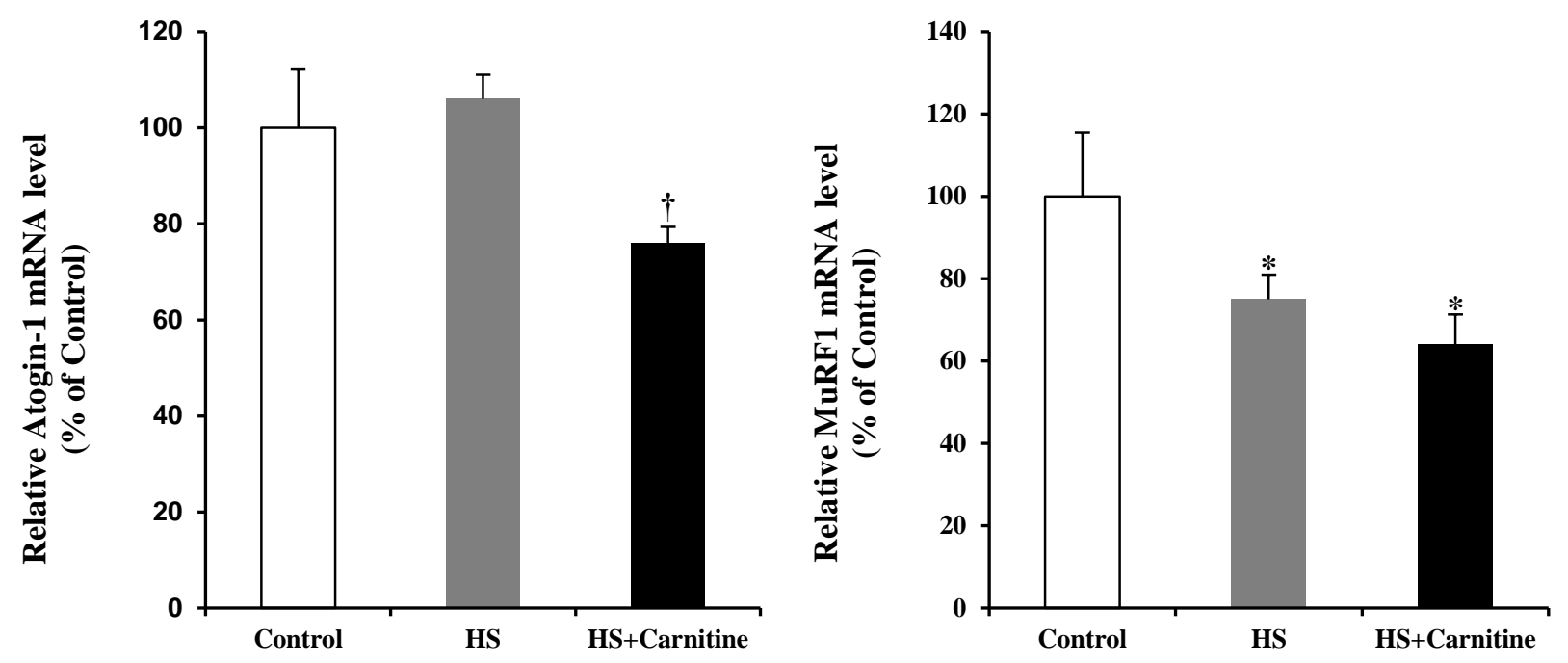
Figure 6
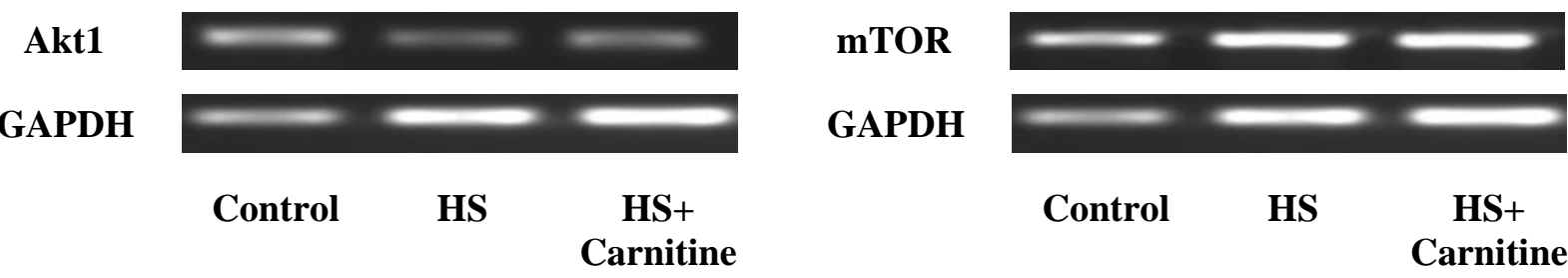
GAPDH

Control

HS HS+ Carnitine

A

B
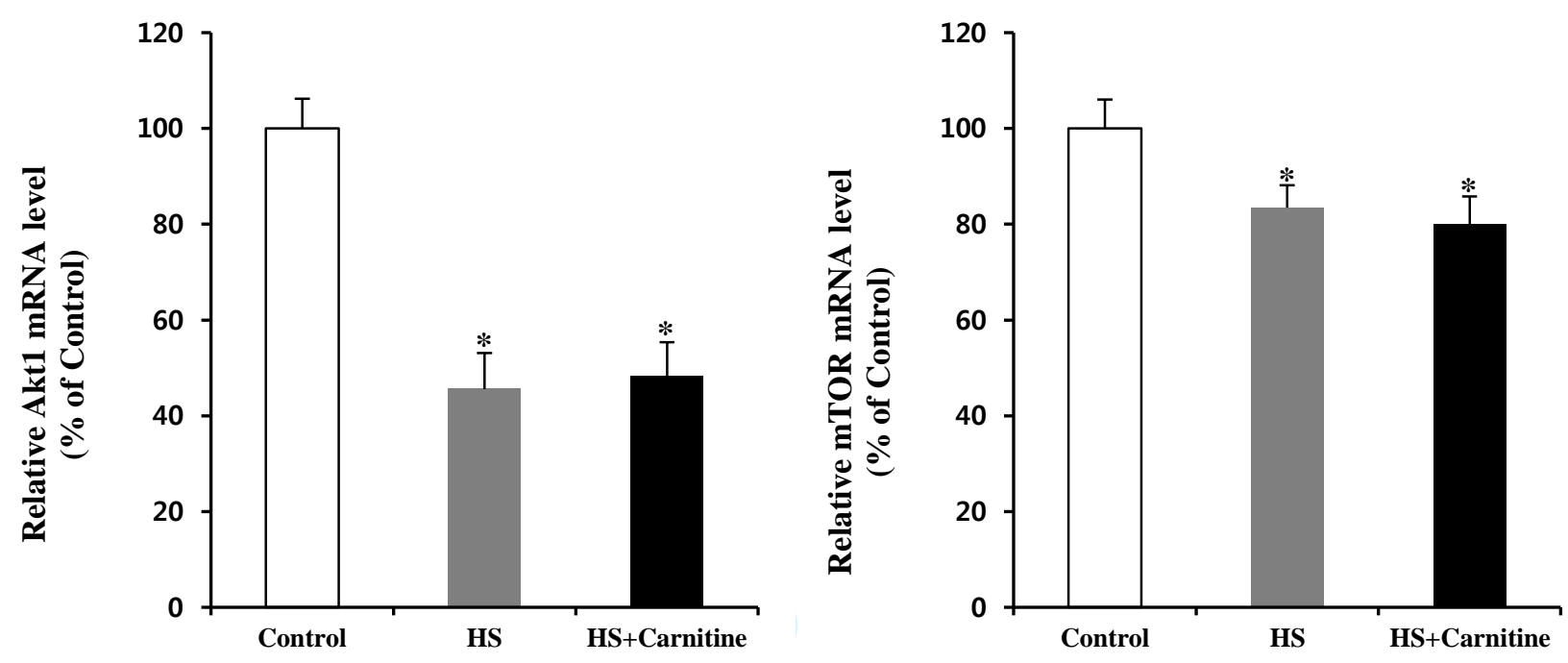
Figure 7

phospho-mTOR

total mTOR

A

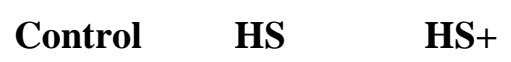

Carnitine

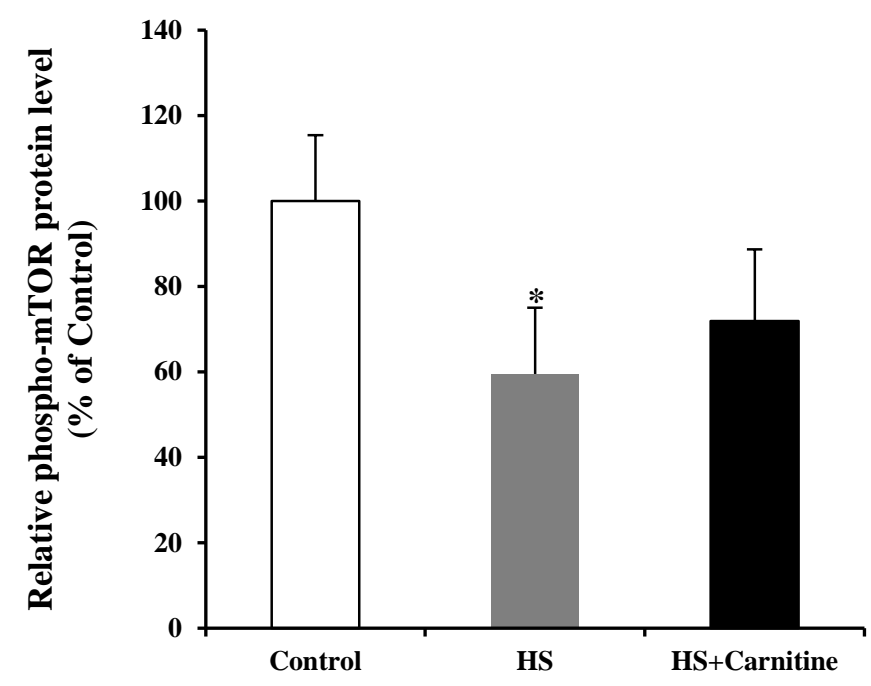

